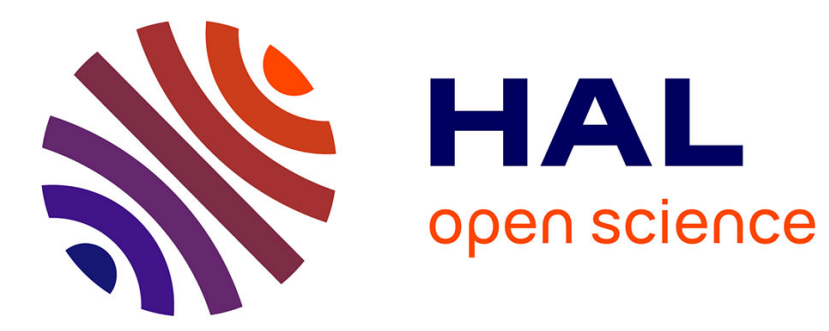

\title{
Generalized decay law for particlelike and wavelike thermal phonons
}

Zhongwei Zhang, Yangyu Guo, Marc Bescond, Jie Chen, Masahiro Nomura, Sebastian Volz

\section{- To cite this version:}

Zhongwei Zhang, Yangyu Guo, Marc Bescond, Jie Chen, Masahiro Nomura, et al.. Generalized decay law for particlelike and wavelike thermal phonons. Physical Review B, 2021, 103 (18), pp.184307. 10.1103/physrevb.103.184307 . hal-03420111

\section{HAL Id: hal-03420111 https://hal.science/hal-03420111}

Submitted on 9 Nov 2021

HAL is a multi-disciplinary open access archive for the deposit and dissemination of scientific research documents, whether they are published or not. The documents may come from teaching and research institutions in France or abroad, or from public or private research centers.
L'archive ouverte pluridisciplinaire HAL, est destinée au dépôt et à la diffusion de documents scientifiques de niveau recherche, publiés ou non, émanant des établissements d'enseignement et de recherche français ou étrangers, des laboratoires publics ou privés. 


\title{
Generalized decay law for particlelike and wavelike thermal phonons
}

\author{
Zhongwei Zhang, ${ }^{1,2,3}$ Yangyu Guo, ${ }^{3}$ Marc Bescond, ${ }^{4}$ Jie Chen $\odot,{ }^{1,2, *}$ Masahiro Nomura, ${ }^{3, \dagger}$ and Sebastian Volz $\odot^{2,4, \$}$ \\ ${ }^{1}$ Center for Phononics and Thermal Energy Science, School of Physics Science and Engineering, \\ Tongji University, 200092 Shanghai, People's Republic of China \\ ${ }^{2}$ China-EU Joint Lab for Nanophononics, Tongji University, 200092 Shanghai, People's Republic of China \\ ${ }^{3}$ Institute of Industrial Science, The University of Tokyo, Tokyo 153-8505, Japan \\ ${ }^{4}$ Laboratory for Integrated Micro and Mechatronic Systems, CNRS-IIS UMI 2820, The University of Tokyo, Tokyo 153-8505, Japan
}

(Received 19 October 2020; revised 25 January 2021; accepted 3 May 2021; published 18 May 2021)

\begin{abstract}
Our direct atomic simulations reveal that a thermally activated phonon mode involves a large population of elastic wave packets. These excitations are characterized by a wide distribution of lifetimes and coherence times expressing particlelike and wavelike natures. In agreement with direct simulations, our theoretical derivation yields a generalized law for the decay of the phonon number taking into account coherent effects. Before the conventional exponential decay due to phonon-phonon scattering, this law introduces a delay proportional to the square of the coherence time. This additional regime leads to a moderate increase of the relaxation times and to a different dependence of phonon relaxation to those. This work opens new horizons in the understanding of the origin and the treatment of thermal phonons.
\end{abstract}

DOI: 10.1103/PhysRevB.103.184307

\section{INTRODUCTION}

The legacy of transport physics establishes that thermal phonons of a given mode can be understood as quasiparticles having the same lifetime and coherence time. Their behaviors are modeled by Boltzmann transport theory and the phonongas model [1,2]. On the other hand, phonons are defined in essence as vibrational waves. Experimental investigations [3-7] demonstrated that the wave nature or the coherence of thermal phonons significantly contributes to thermal transport. For example, Maire et al. [5] could tune thermal conduction by using this coherence in silicon phononic crystals.

Previous studies also demonstrated that the particlelike and wavelike thermal phonons are coexisting at elevated temperature $[4,4,8-10]$. In epitaxial oxide superlattices, Ravichandran et al. [4] experimentally observed this mechanism and the predominant behavior depends on temperature and period. However, state-of-the-art theories are failing in simultaneously capturing both wave and particle pictures $[1,2,11]$. Alternative methods still miss a direct representation of thermal phonon excitations [12-15], which is the case when determining phonon lifetimes from anharmonic lattice dynamics [16-18] and in experimental measurements $[19,20]$. Recently, several groups [21,22] proposed models to consider the coherence between different thermal phonon modes, and significant effects on thermal transport are observed in glasses or complex crystals; however, the intrinsic coherence or wavelike behavior of thermal phonons still could not be revealed.

In this paper, we track the real phonon dynamics and extract temporal coherence times and lifetimes by using the

\footnotetext{
*jie@tongji.edu.cn

${ }^{\dagger}$ nomura@iis.u-tokyo.ac.jp

${ }^{\ddagger}$ volz@iis.u-tokyo.ac.jp
}

wavelet transform of the atomic trajectories during an equilibrium molecular dynamic (MD) simulation. We find that a thermally activated single phonon mode involves a large distribution of excitations with a broad range of coherence times and lifetimes. A theory is proposed to establish the relationship between coherence times and lifetimes, which reveals the unexpected impact of long coherence times on phonon relaxation and phonon decay. A generalized decay law is proposed by simultaneously including particlelike and wavelike behaviors of thermal phonons. These conclusions provide insights into the reality of thermally activated phonon modes and their intrinsic wavelike and coherence behaviors.

\section{SIMULATION}

\section{A. Direct simulation of phonon dynamics}

Fourier transform has been widely used in phonon-related analysis, since it provides the natural function basis of phonons in the form of monochromatic plane waves $e^{-i(\omega t-\mathbf{k} \cdot \mathbf{r})}$ $[23,24]$, where the plane wave comprises temporal $t$ and spatial $\mathbf{r}$ dependences. Energy transport, however, is understood as a spatially and temporally localized wave packet [25-28]. To describe this transport, Baker et al. [29] and Shiomi and Maruyama [30] proposed wavelet transforms to investigate the propagation of phonon energy, in which the functions of the basis take the form of a Gaussian wave packet. Modifying this wavelet transform approach and focusing on the temporal extension information, the expression of the normalized phonon wavelet basis is written as

$$
\psi_{\omega_{\mathbf{k s} s}, t_{0}, \Delta_{\mathbf{k} s}}(t)=\pi^{-1 / 4} \Delta_{\mathbf{k} s}^{-1 / 2} e^{\left[i \omega_{\mathbf{k} s}\left(t-t_{0}\right)\right]} e^{\left[-1 / 2\left(t-t_{0} / \Delta_{\mathbf{k} s}\right)^{2}\right]},
$$

where $\omega_{\mathbf{k} s}$ is the angular frequency of mode $\mathbf{k} s$, and $\Delta_{\mathbf{k} s}$ defines the wave-packet duration. $t$ corresponds to the time variable, and $t_{0}$ to the position of highest amplitude in the wave packet, and also corresponds to the time evolution in 
(a)
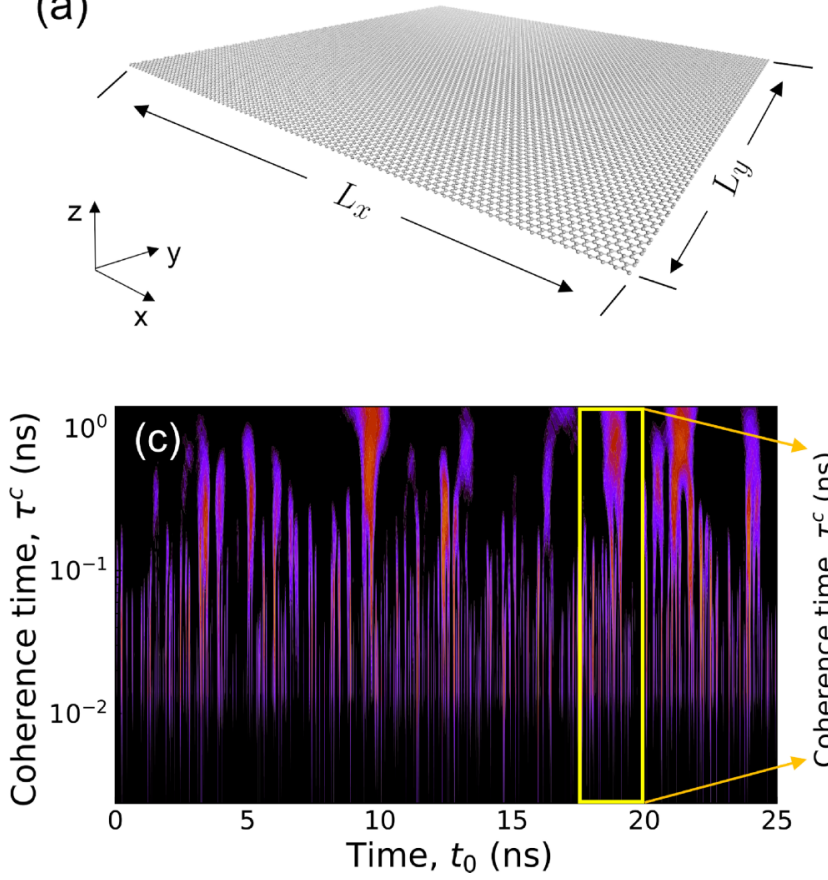
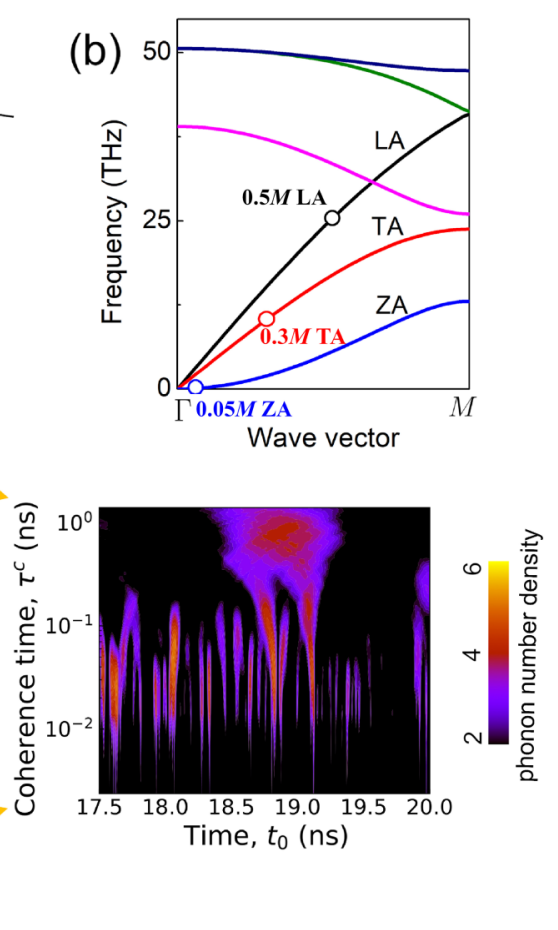

FIG. 1. Evolution time and coherence time dependent phonon number. (a) Schematic figure of a suspended single-layer graphene. The domain of the graphene system is set to $L_{x}=L_{y} \approx 100 \mathrm{~nm}$. The convergence of the results with size was carefully checked. (b) Phonon dispersion of single-layer graphene along $\Gamma(0,0,0) \rightarrow M(0.5,0,0)$. The circles specify the three analyzed modes: $0.05 M$ ZA $(\mathrm{ZA}$ mode at the $k$ point of $0.05 \times(0.5,0,0)), 0.3 M$ TA, and $0.5 M$ LA. (c) Evolution time $\left(t_{0}\right)$ and coherence time $\left(\tau^{c}\right)$ dependent phonon number density of the 0.05M ZA mode at room temperature. The right-hand side inset highlights the 17.5-20.0 ns interval of (c).

the wavelet space. Inside the wave packet, plane waves are in phase; the $\Delta_{\mathbf{k} s}$ term in Eq. (1) is thus a measure of the temporal coherence of thermal phonons. Here, we define the wave packet full width at half maximum (FWHM) as the coherence time $\tau_{\mathbf{k} s}^{c}=2 \sqrt{2 \ln 2} \Delta_{\mathbf{k} s}$. This basis leads to the following wavelet transform:

$$
\Lambda\left(\omega_{\mathbf{k} s}, t_{0}, \tau_{\mathbf{k} s}^{c}\right)=\int \psi_{\omega_{\mathbf{k s} s}, t_{0}, \tau_{\mathbf{k} s}^{c}}(t) F(t) d t,
$$

where $F(t)$ denotes the time dependent dynamical quantity, which is chosen as the phonon modal velocity, $\frac{1}{a} \sum_{b, l}\left[\dot{\mathbf{u}}_{b l}(t) \cdot \mathbf{e}_{b}^{*}(\mathbf{k}, s) \times \exp \left(i \mathbf{k} \cdot \mathbf{R}_{0 l}\right)\right]$, where $\dot{\mathbf{u}}_{b l}(t)$ denotes the velocity of the $b$ th atom in the $l$ th unit cell at time $t, a$ the number of cells, $\mathbf{e}^{*}(\mathbf{k}, s)$ refers to the complex conjugate of the eigenvector of mode $\mathbf{k} s$, and $\mathbf{R}_{0 l}$ is the equilibrium position of the $l$ th unit cell. As a control calculation, we validated the relevance of wavelet transform to study phonon properties, i.e., eigenfrequency, temporal coherence, and creation/annihilation of wave packets (see Appendix A for details). We highlight that the wavelet transform successfully provided the same results as those predicted by commonly used spectral energy density (SED) analysis $[23,31]$. The SED is in agreement with the single-mode relaxation time (SMRT) approximation. The wavelet transform is a further expansion of SED (see Fig. 7 in Appendix A).

We use classical MD simulations to obtain the real phonon dynamics in the graphene system shown in Fig. 1(a) at elevated temperatures. Compared to other theories, i.e., Grüneisen parameter based Klemens model or anharmonic lattice dynamics [32-34], phonon-phonon scattering is fully excited in MD simulations. The $\mathrm{C}-\mathrm{C}$ interaction is modeled by the optimized Tersoff potential [35]. All MD simulations are performed by implementing the graphics processing units molecular dynamics (GPUMD) package [36] with a time step of 0.35 fs. To extract reliable time dependent information, five MD simulations with time duration of $25 \mathrm{~ns}$ were carried out. From Eq. (2), the time dependent phonon number at a given coherence time, here called phonon number density, $N\left(\omega_{\mathbf{k} s}, t_{0}, \tau_{\mathbf{k} s}^{c}\right)$ can be calculated as $N\left(\omega_{\mathbf{k} s}, t_{0}, \tau_{\mathbf{k} s}^{c}\right)=$ $\frac{1}{2} m\left|\Lambda\left(\omega_{\mathbf{k} s}, t_{0}, \tau_{\mathbf{k} s}^{c}\right)\right|^{2} / \hbar \omega_{\mathbf{k} s}$, where $m$ refers to the mass of the carbon atom. Wavelet transformation finally provides the mode energy distribution in evolution time and in coherence time. The time dependent phonon number reads $N\left(\omega_{\mathbf{k} s}, t_{0}\right)=$ $\sum_{\tau_{\mathbf{k} s}^{c}} N\left(\omega_{\mathbf{k} s}, t_{0}, \tau_{\mathbf{k} s}^{c}\right)$. This coherence time dependent phonon number obtained from simulation corresponds to the phonon number in Eq. (8) because only one term of the sum contributes at a given time $t_{0}$ due to the presence of a single wave packet at this same time in the mode.

\section{B. Coherence time distribution}

Figure 1(c) reports the phonon number density $N\left(\omega_{\mathbf{k} s}, t_{0}, \tau_{\mathbf{k} s}^{c}\right)$ derived from Eqs. (1) and (2) for the $0.05 M$ ZA mode. Dark areas indicate the absence of phonon energy and the brighter ones represent the apparition of phonon wave packets. While common understanding stipulates a unique coherence time per mode, we uncover a distribution of coherence times instead. In addition, this distribution is varying with time. The right-hand side inset of Fig. 1(c) displays a $2.5 \mathrm{~ns}$ time interval of the phonon number density 


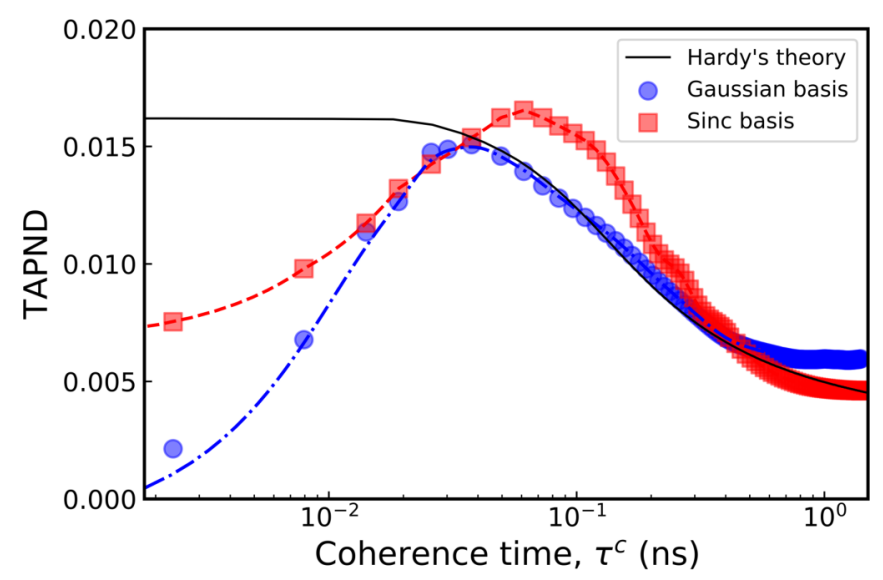

FIG. 2. Time-averaged phonon number density (TAPND) as a function of coherence time. The density distribution is calculated either with a Gaussian function basis (blue circles) or with a sinus cardinal function basis (red squares), for the 0.05M ZA mode at room temperature. The solid line is a trend based on Hardy's theory [37].

history. It turns out that phonon coherence times of the studied mode cover two orders of magnitude. Subpopulations manifest very long coherence $\left(\tau_{\mathbf{k} s}^{c}>300 \mathrm{ps}\right)$, i.e., a wavelike feature, whereas others with shorter coherence times $\left(\tau_{\mathbf{k} s}^{c}<\right.$ $10 \mathrm{ps}$ ) can be assimilated to particlelike excitations.

The wave-packet distribution can be further investigated by building the time-averaged phonon number density (TAPND) versus coherence time $D\left(\omega_{\mathbf{k} s}, \tau_{\mathbf{k} s}^{c}\right)=\frac{1}{N_{t_{0}}} \sum_{t_{0}} \frac{N\left(\omega_{\mathbf{k s}}, t_{0}, \tau_{\mathbf{k}}^{c}\right)}{\sum_{\tau_{\mathbf{k} s}^{c}}^{c}\left(\omega_{\mathbf{k} s}, t_{0}, \tau_{\mathbf{k s}}^{c}\right)}$, reported in Fig. 2, where $N_{t_{0}}$ denotes the number of terms in the sum. Interestingly, wave packets follow a unimodal distribution as a function of coherence time, indicating the predominance and the limits in duration of a subpopulation centered at $\tau^{c} \sim 40 \mathrm{ps}$. The TAPND was not observed before, only its average on $\tau^{c}$ obtained by other methods [8]. Previously, the founding work of Hardy [37] mentioned that the TAPND of a given mode $\mathbf{k}$ should be distributed along an

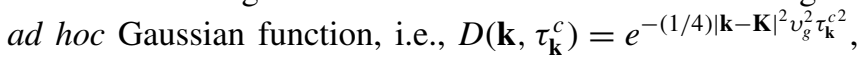
where $\mathbf{K}$ denotes the wave vector of a mode interfering with mode $\mathbf{k}$ and $v_{g}$ corresponds to the group velocity of mode $\mathbf{k}$. Hardy's prediction fairly agrees with our analysis in the long wave-packet range as highlighted by Fig. 2. Disagreement appears in the low coherence time region, presumably because coherence time has to be larger than mode period, which is not taken into account by Hardy's proposition. While a full confirmation for all modes is unreachable, a similar unimodal distribution of the TAPND is also observed for other graphene modes as well as in bulk silicon (see Appendix B for details).

\section{Phonon decay}

The autocorrelation of the fluctuations of the phonon number density provides a basis for comparison with the conventional description of the phonon mode decay. The autocorrelation function $C\left(t, \tau_{\mathbf{k} s}^{c}\right)$ as outcome of our simulations is calculated as

$$
C\left(t, \tau_{\mathbf{k} s}^{c}\right)=\frac{\left\langle\Delta N\left(t, \tau_{\mathbf{k} s}^{c}\right) \Delta N\left(0, \tau_{\mathbf{k} s}^{c}\right)\right\rangle}{\left\langle\Delta N\left(0, \tau_{\mathbf{k} s}^{c}\right) \Delta N\left(0, \tau_{\mathbf{k} s}^{c}\right)\right\rangle},
$$

where $\Delta N\left(t_{0}, \tau_{\mathbf{k} s}^{c}\right)=N\left(t_{0}, \tau_{\mathbf{k} s}^{c}\right)-\left\langle N\left(t_{0}, \tau_{\mathbf{k} s}^{c}\right)\right\rangle_{t_{0}}$. For small coherence time wave packets, a rapid decay to zero is observed in Fig. 3(a) and as coherence time increases, the correlation time does also. In the established knowledge, phonon number decay should be described by an exponential function, $\sim e^{-t / \tau_{\mathbf{k s}}^{l}}$, where $\tau_{\mathbf{k} s}^{l}$ is the lifetime for mode $\mathbf{k} s$, when the SMRT approximation [18] applies. Unfolding this decay versus coherence times, the correlations $C\left(t, \tau_{\mathbf{k} s}^{c}\right)$ clearly deviate from the exponential trend. This deviation also intensifies with coherence time as illustrated by Fig. 3(b). Those results evidence the failure of the SMRT approximation in capturing wave-packet coherence. Note that the effect of this latter differs from the influence of collective mode coupling shown in graphene $[11,38]$ that also invalidates SMRT but preserves the exponential relaxation [11].

\section{THEORY}

\section{A. Coherence time dependent phonon number}

Previously, Hardy [37] demonstrated that the harmonic energy-flux operator $\mathbf{S}$ can be expressed as

$$
\mathbf{S}=\frac{1}{V} \sum_{\mathbf{k} s} N_{\mathbf{k} s} \hbar \omega_{\mathbf{k} s} v_{\mathbf{k} s}
$$

We will use the $\mathbf{k} s=\lambda$ notation in the following as the mode wave vector and branch will have to be distinguished. Note that Eq. (4) clearly highlights that the phonon energy, in any form, is transported at the group velocity $[27,28]$. When rewriting Eq. (4) in terms of the classical coordinates and considering the fluctuation of the phonon energy, the time dependent phonon number becomes (see Appendix C)

$$
N_{\mathbf{k} s}(t)=\frac{1}{\hbar} \sum_{\mathbf{k}^{\prime}} p_{\mathbf{k} s}(t) q_{\mathbf{k}^{\prime} s}^{*}(t),
$$

where $p_{\mathbf{k} s}(t)$ and $q_{\mathbf{k} s}(t)$ denote the time dependent normal mode momentum and displacement for mode $\mathbf{k} s . *$ indicates the conjugate form. Here, we ignored the terms $s \neq s^{\prime}$ that are rapidly oscillating and yield negligible time average. Because of phonon-phonon scattering, normal mode coordinate decays with time, i.e., $q_{\mathbf{k} s}(t)=q_{\mathbf{k} s}(0) e^{-\Gamma_{\mathbf{k} s} t-i \omega_{\mathbf{k} s} t}$, where $\Gamma_{\mathbf{k} s}$ represents the mode linewidth, involved in the SMRT approximation $[16,28,39]$. Consequently, the particlelike behavior of phonons is firstly included in the derivation. Accordingly, the time dependent momentum can be expressed as $p_{\mathbf{k} s}(t)=$ $p_{\mathbf{k} s}(0) e^{-\Gamma_{\mathbf{k s}} t-i \omega_{\mathbf{k} s} t}$. Note that for now, the initial time $t=0$ is arbitrarily taken as that of maximum amplitude. This assumption will, however, become unnecessary when deriving the autocorrelation functions. It turns out that the time evolution of the phonon number is obtained as

$$
N_{\mathbf{k} s}(t)=\sum_{\mathbf{k}^{\prime}} \xi_{\mathbf{k k}^{\prime} s} e^{-\gamma_{\mathbf{k} \mathbf{k}^{\prime} s} t-i \Delta \omega_{\mathbf{k} \mathbf{k}^{\prime} s} t},
$$

where $\quad \xi_{\mathbf{k k}^{\prime} s}=p_{\mathbf{k} s}(0) q_{\mathbf{k}^{\prime} s}^{*}(0) / \hbar, \quad \gamma_{\mathbf{k k}^{\prime} s}=\Gamma_{\mathbf{k} s}+\Gamma_{\mathbf{k}^{\prime} s}, \quad$ and $\Delta \omega_{\mathbf{k k}^{\prime} s}=\omega_{\mathbf{k} s}-\omega_{\mathbf{k}^{\prime} s}$. It should be noted that the summation over $\mathbf{k}^{\prime}, \mathbf{k} \neq \mathbf{k}^{\prime}$, can be understood as the interference of plane waves defined by $\mathbf{k} s$ and $\mathbf{k}^{\prime} s$, which are forming wave packets. As will be confirmed in later simulation results, we shall assume that a single wave packet appears at a given time. Considering that this wave packet is resulting from a specific 

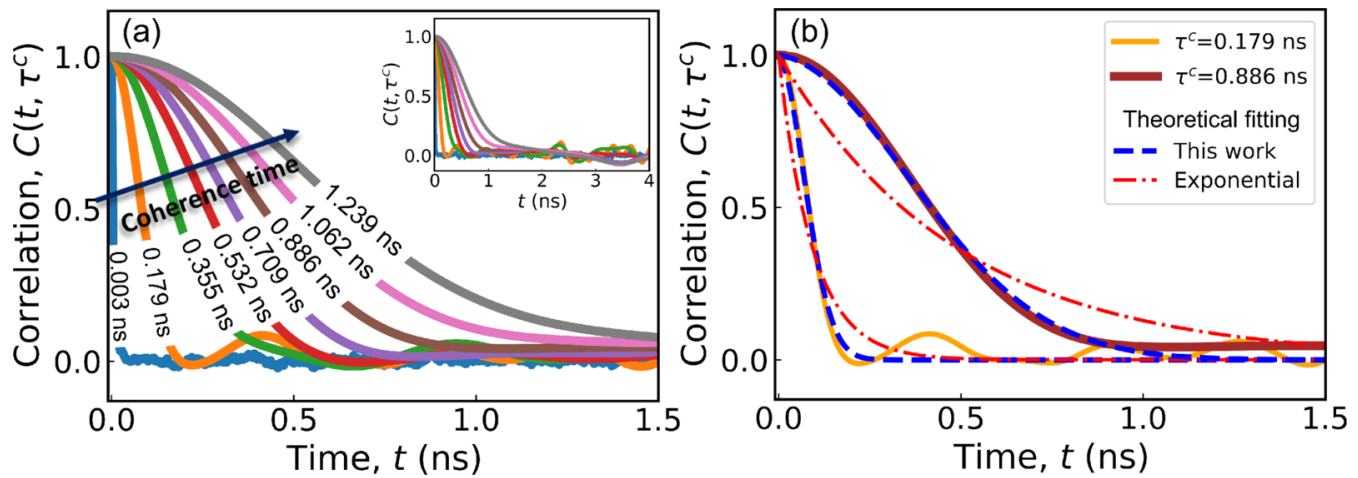

FIG. 3. Fitting of the phonon number density decay. (a) Phonon number density correlation function $C\left(t, \tau^{c}\right)$ versus correlation time $t$ for several coherence times $\tau^{c}$. The inset shows the correlation function running over $4 \mathrm{~ns}$. (b) Fitting of the MD correlation function (continuous lines) with the conventional exponential decay theory (dash-dotted lines) and the theory of this work (dashed lines). The phonon decay is for the $0.05 \mathrm{M} \mathrm{ZA}$ mode at room temperature.

and restricted frequency interval $\left[\omega_{\mathbf{k} s}-\frac{\Omega_{\mathbf{k} s}}{2}, \omega_{\mathbf{k} s}+\frac{\Omega_{\mathbf{k} s}}{2}\right]$ where density of states and linewidth are nearly constant, a further step can be taken,

$$
N_{\mathbf{k} s}(t) \approx \bar{\xi}_{\mathbf{k} s} e^{-\bar{\gamma}_{\mathbf{k} s} t} \bar{g}\left(\omega_{\mathbf{k} s}\right) \int_{-\Omega_{\mathbf{k} s} / 2}^{+\Omega_{\mathbf{k} s} / 2} e^{-i \Delta \omega_{\mathbf{k} \mathbf{k}^{\prime} s} t} d \Delta \omega_{\mathbf{k} \mathbf{k}^{\prime} s}
$$

where $\bar{\xi}_{\mathbf{k} s}$ and $\bar{\gamma}_{\mathbf{k} s}$ correspond to the averaged properties over the wave-packet frequency interval and $\bar{g}\left(\omega_{\mathbf{k} s}\right)$ refers to the density of states. We shall also highlight that Eq. (7) is introducing the key quantity of $\Omega_{\mathbf{k} s}$, which will be shown to be proportional to the reverse of the coherence time. Due to the previously mentioned assumption of "single wave packet at a time," we emphasize that a single value of $\Omega_{\mathbf{k} s}$ can indeed be involved at a given time $t$ in the expression of the mode phonon number. The integral of Eq. (7) can be seen as a combination of plane waves [40] and its estimation yields (see Appendix C)

$$
N_{\mathbf{k} s}(t)=2 \bar{\xi}_{\mathbf{k} \mathbf{s}} \bar{g}\left(\omega_{\mathbf{k} s}\right) e^{-\bar{\gamma}_{\mathbf{k} s} s} \frac{\sin \pi \Omega_{\mathbf{k} s} t}{t} .
$$

This derivation indicates that at time $t$ a phonon wave packet with properties $\bar{\gamma}_{\mathbf{k} s}$ and $\Omega_{\mathbf{k} s}$ is contributing to the phonon number $N_{\mathbf{k} s}(t)$. This phonon wave packet should take the form of a sinus cardinal function, which is close to the Gaussian function but with a longer tail (see Fig. 9 in Appendix $C$ ). Those two functions indeed have the same form in the vicinity of the origin, and other arguments provided by later simulations will also confirm the approximation of a sinus cardinal by a Gaussian.

\section{B. Generalized phonon decay law}

To analytically predict the coherence correction in the phonon decay, we then obtain the autocorrelation of this coherence time dependent phonon number $[41,42]$ (see Appendix $\mathrm{D}$ for complete derivation), which takes the final following form:

$$
C\left(t, \tau_{\mathbf{k} s}^{c}\right)=e^{-t / 2 \tau_{\mathbf{k} s}^{l}} e^{-4 \ln 2\left[t^{2} / \tau_{\mathbf{k} s}^{c}{ }^{2}\right]},
$$

where the two decay terms of the right-hand side correspond to the two distinct behaviors of thermal phonons, i.e., the particlelike and the wavelike ones. The particlelike part remains an exponentially decaying function, and the second one appears as a quadratic Gaussian term.

The autocorrelation of Eq. (9) can be further factorized into

$$
C\left(t, \tau_{\mathbf{k s} s}^{c}\right)=e^{-4 \ln 2\left[\left(t+\tau_{w}\right)^{2} / \tau_{\mathbf{k s}}^{c}\right]} e^{\tau_{w} / 4 \tau_{\mathbf{k s}}^{l}},
$$

where $\tau_{w}=\tau_{\mathbf{k} s}^{c 2} /\left(\tau_{\mathbf{k} s}^{l} 16 \ln 2\right)$ evaluates the effect of coherence on phonon decay. This latter equation reveals the general form of a Gaussian decay for the phonon number in which the very short time regime $t \ll \tau_{w}$ yields strong correlation $C\left(t, \tau_{\mathbf{k} s}^{c}\right) \approx 1$ as seen in Fig. 3(a) (line with $\tau_{\mathbf{k} s}^{c}=1.239 \mathrm{ns,}$ $t<0.5 \mathrm{~ns}$ ), and intermediate times $t<\tau_{w}$ lead back to the usual phonon-phonon scattering decay $C\left(t, \tau_{\mathbf{k} s}^{c}\right)=e^{-t / 2 \tau_{\mathbf{k s}}^{l}}$ after Taylor expansion. The longer time interval $t>\tau_{w}$ brings a Gaussian decay $C\left(t, \tau_{\mathbf{k s} s}^{c}\right)=e^{-4 \ln 2 t^{2} / \tau_{\mathbf{k s}}^{c} e^{2}} e^{\tau_{w} / 4 \tau_{\mathbf{k s}}^{l}}$. The first short time regime is due to the buildup of the wave packet and is delaying the relaxation compared to the conventional exponential decay. From Eq. (9), we can simultaneously obtain $\tau_{\mathbf{k} s}^{l}$ and $\tau_{\mathbf{k} s}^{c}$ for a given mode by fitting the autocorrelations of Fig. 3. As shown in Fig. 3(b), the disagreement between MD results and the exponential fitting is larger compared to the one resulting from the predictions of Eq. (9).

\section{LIFETIME AND COHERENCE TIME}

Figure 4 shows the fitted lifetimes and coherence times according to Eq. (9). Obviously, for short wave packets, i.e., small coherence times, phonons exhibit prominent particlelike behaviors but with long lifetimes. As coherence time increases, the time spread of the phonon wave packets becomes longer, with pronounced wave behaviors as illustrated in Fig. 4(b). The lifetime follows a nonmonotonic dependence on coherence time depicted in Fig. 4(a) with a transition point around the coherence time of $0.45 \mathrm{~ns}$. Larger phonon wave packets $\left(\tau_{\mathbf{k} s}^{c}>0.45 \mathrm{~ns}\right)$ possess shorter lifetimes, while a set of wave packets at $\sim 0.45 \mathrm{~ns}$ survive on longer periods. Note that phonons are almost monotonically transiting from the particlelike nature to the wavelike nature with increasing coherence time as shown by the time ratio in Fig. 4(b). In addition, this transition is also frequency dependent as low frequency phonons in the $0.05 \mathrm{M}$ ZA mode display wavelike behaviors at shorter coherence times. 

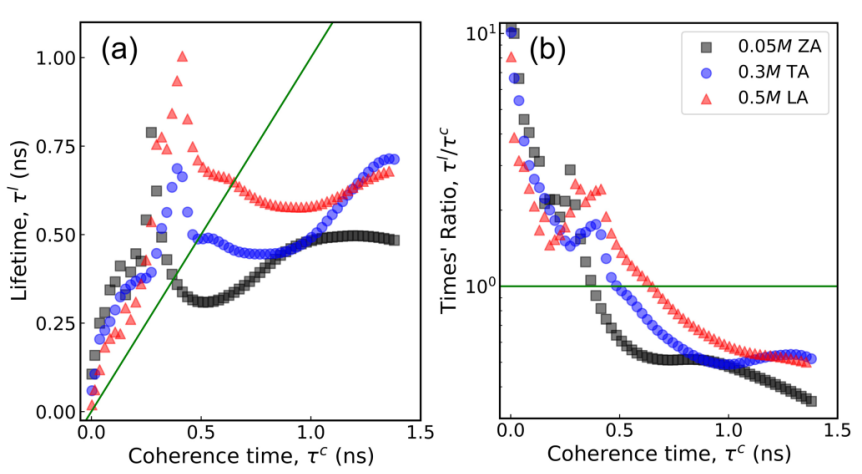

FIG. 4. Lifetime and coherence time in graphene. (a) Fitted lifetime and coherence time in graphene, and (b) ratio between lifetime and coherence time for modes $0.05 M \mathrm{ZA}, 0.3 M \mathrm{TA}$, and $0.5 M \mathrm{LA}$ at room temperature. The green line indicates the lifetimes $=$ coherence times condition. Above the line, phonons show particlelike nature properties with lifetimes $>$ coherence times. In contrast, below the line, phonons have wavelike nature properties with lifetimes $<$ coherence times.

The influence of temperature on the wave packet coherence time and lifetime was also studied in Fig. 5. As temperature increases, phonon-phonon scattering intensifies and the particle nature of phonons, i.e., the contribution of short coherence time wave packets, becomes more obvious. As reported in Fig. 5(a), this trend is evidenced by a shift of the density peak to lower coherence times and by the gradual suppression of long wave packets. Moreover, the decrease of phonon lifetimes with temperature can also be observed in the reduction of the times' ratio $\tau^{l} / \tau^{c}$ with temperature.

\section{CONCLUSION}

The wavelet transform calculations and the proposed phonon decay theory [Eq. (10)] prove that a single thermally activated phonon mode includes excitations with a broad range of lifetimes and coherence times. The commonly used SMRT theory is based on a single phonon lifetime per mode and the assumption of exponential decay to fit the phonon number autocorrelation function. This paper proposes a step forward, by unfolding the phonon number over the coherence times and rewriting the autocorrelation function of this number as a combination of coherence time dependent correlation functions.

Beyond the here investigated phonon propagation, the wavelike behavior of phonons should also be critical in the treatment of surface phonon scattering and propagation in nanostructures, superlattices, and interfaces. Uncovering here the unexpected content of thermal phonon excitations leads to new perspectives in the understanding of thermal coherence and the essence of a blackbody in nanostructures as well as in bulk materials.

\section{ACKNOWLEDGMENTS}

This project is supported in part by grants from the National Natural Science Foundation of China (Grants No. 12075168 and No. 11890703), and Science and Technology Commission of Shanghai Municipality (Grants No. 19ZR1478600 and No. 18JC1410900). This work is partially supported by CREST JST (Grant No. JPMJCR19Q3). Z.Z. gratefully acknowledges financial support from China Scholarship Council.

\section{APPENDIX A: VALIDATION OF WAVELET TRANSFORM}

A benchmark is proposed to illustrate the reliability of the wavelet transform approach in the study of phonon properties. Firstly, artificial wave packets are generated with forms based on Eq. (1) of the main text. As shown in Fig. 6(a), two artificial wave packets are triggered at times 30 and 80 ps. Other parameters are set as $1 \mathrm{THz}$ eigenfrequency and $6 \mathrm{ps}$ FWHM. Usually, the spectral energy density method is used to study phonon properties, especially phonon lifetime and
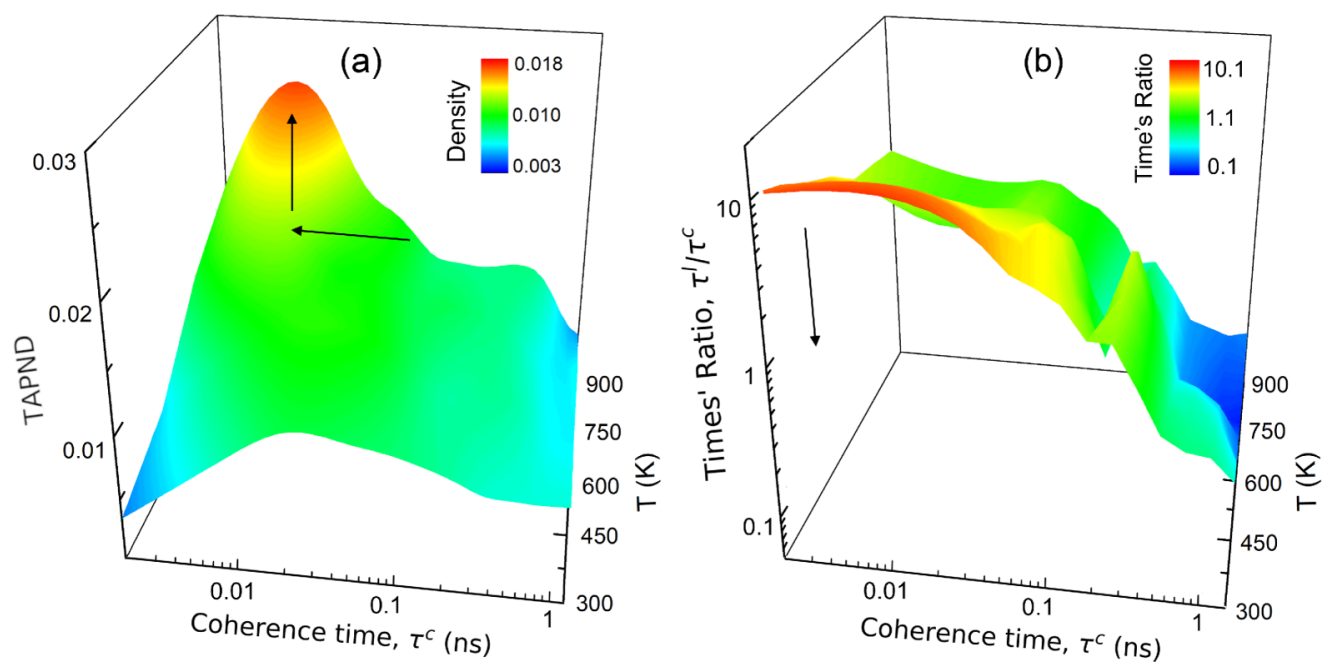

FIG. 5. Temperature effect on the phonon number density. (a) Time-averaged phonon number density (TAPND) $D\left(\omega, \tau_{c}\right)$ versus coherence time $\tau^{c}$ as a function of temperature for the $0.05 M$ ZA mode. The arrows indicate the shift of the peak towards lower $\tau^{c}$ values and a higher density with increasing temperature. (b) Times' ratio $\tau^{l} / \tau^{c}$ versus coherence time $\tau^{c}$ as a function of temperature for the $0.05 M$ ZA mode. The arrow indicates the shift of the ratio $\tau^{l} / \tau^{c}$ towards lower densities with temperature. 

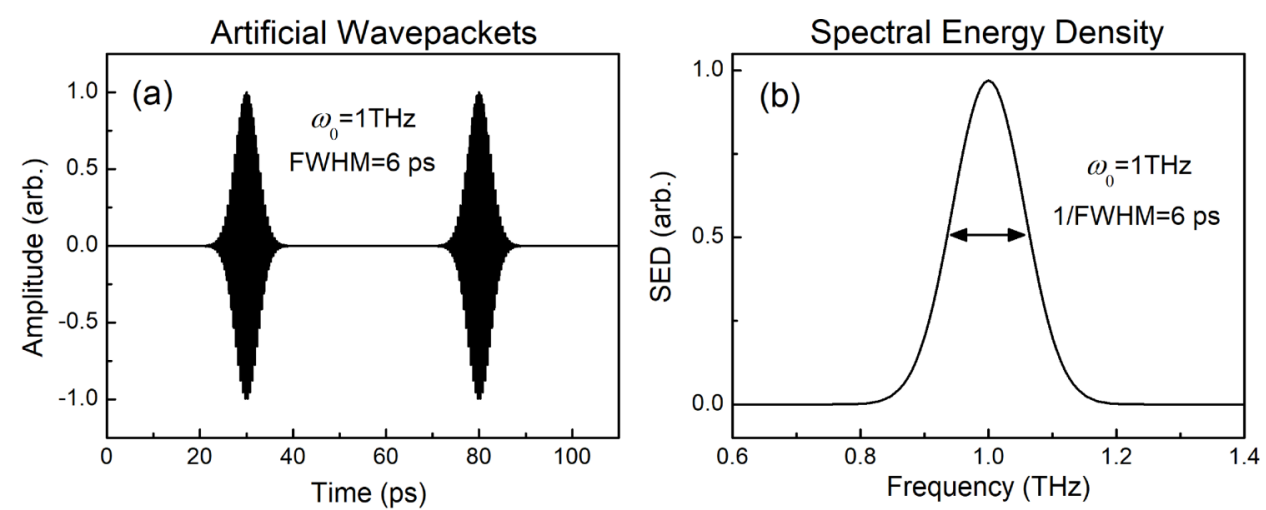

Wavelet Transform Results

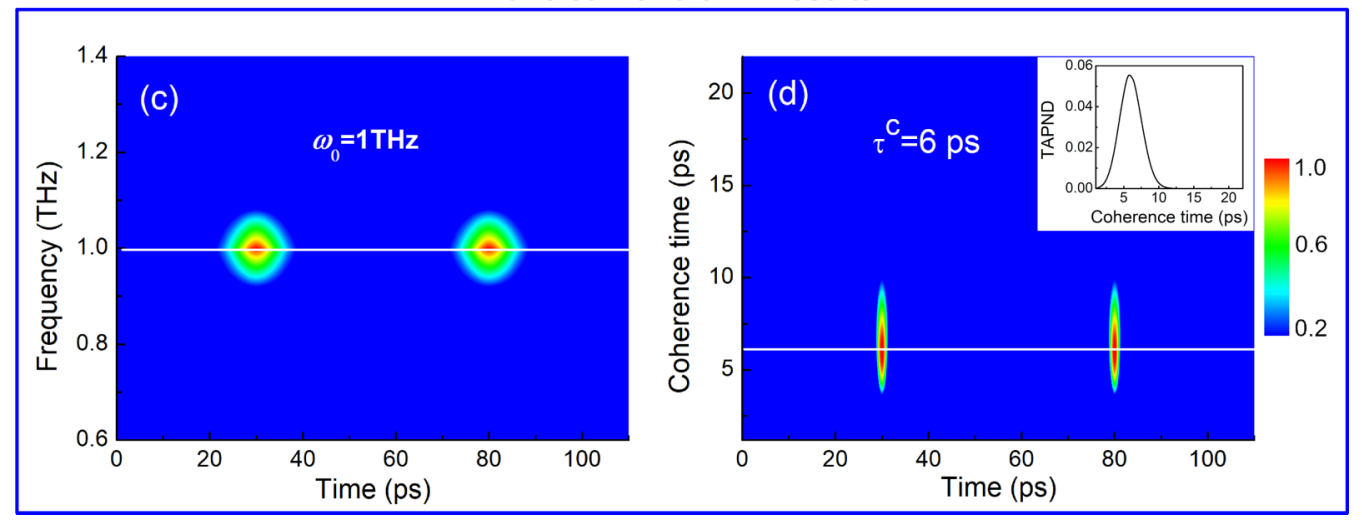

FIG. 6. Validation of the wavelet transform in the study of phonon properties. (a) Two artificial wave packets generated based on Eq. (1) with frequency $1 \mathrm{THz}$ and full width at half maximum (FWHM) 6 ps, triggered at times 30 and 80 ps. (b) Spectral energy density (SED) of the artificial wave packets in (a). (c) Frequency versus evolution time obtained from wavelet transform. (d) Coherence time versus evolution time obtained from wavelet transform. The inset of (d) is the time-averaged phonon number density (TAPND) versus coherence time. The color bar indicates the normalized phonon number density.

eigenfrequency [23]. The calculated time in SED, which is always expressed as the reverse of the SED FWHM, is also the temporal spreading of the wave packet and thus the lifetime. By using the SED method, we precisely retrieve the same set of parameters as those of the artificial wave packets.

By varying frequency and performing the wavelet transform in Eqs. (1) and (2), Fig. 6(c) reports the frequency versus evolution time for the artificial wave packets. Obviously, we can accurately obtain the frequency, the creation, and annihilation times of wave packets from the input information. On the other hand, by varying the coherence time in Eq. (1) and performing the wavelet transform, we can extract the information of coherence time versus evolution time. As shown in Fig. 6(c), the obtained mean coherence time agrees well with the initial set of parameters. Moreover, the inset of Fig. 6(d) shows the TAPND as a function of coherence time. Firstly, the peak position at $\sim 6$ ps agrees well with the set of coherence time in the artificial wave packets. In addition, the broadening of this peak of $3.9 \mathrm{ps}$ is several orders of magnitude smaller than the studied coherence time of phonon wave packets in graphene, indicating a sufficient resolution in coherence time of the wavelet transform. Therefore it can be concluded that the wavelet transform can precisely obtain the phonon properties, i.e., eigenfrequency, coherence time, and also wave-packets creation and annihilation dynamics, which agrees well with the commonly used SED method.
The SED analysis is based on the SMRT approximation theory, in which phonon decay follows an exponential function, and also Lorentz function in the frequency space $[16,23]$. The SED analysis is always performed by the Fourier transform with plane-wave basis. Differently, in the wavelet transform, a Gaussian term is further added to the plane-wave basis to describe the coherence dimension [see Eq. (1)]. The treatment can be considered as an expansion of the normal SMRT approximation to the temporal coherence dimension. After the expansion, the temporal information of phonon wave packets can be obtained, including time dependent dynamics and also the coherence distribution of wave packets as illustrated in Fig. 7.

\section{APPENDIX B: OBSERVATIONS IN SILICON}

Figure 8 shows that the $0.05 M$ TA mode of bulk silicon displays a similar TAPND unimodal distribution as in graphene.

\section{APPENDIX C: HEAT FLUX AND TIME DEPENDENT PHONON NUMBER}

In the following, we focus on the harmonic energy flux. Thus, the simplified energy-flux operator for mode $\mathbf{k} s$ is defined as in Ref. [37]:

$$
\mathbf{S}=-\frac{1}{2 V} \sum_{\mathbf{k} \mathbf{k}^{\prime}, s s^{\prime}}\left(a_{\mathbf{k} s}+a_{-\mathbf{k} s}^{\dagger}\right) \times\left(a_{\mathbf{k}^{\prime} s^{\prime}}-a_{-\mathbf{k}^{\prime} s^{\prime}}^{\dagger}\right) \hbar \omega_{\mathbf{k} s} v_{\mathbf{k} s},
$$




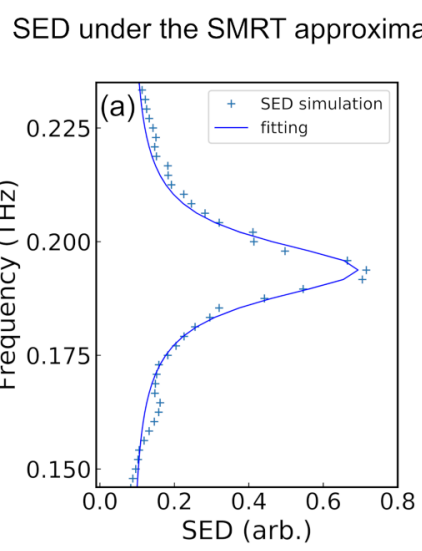

Wavelet transform
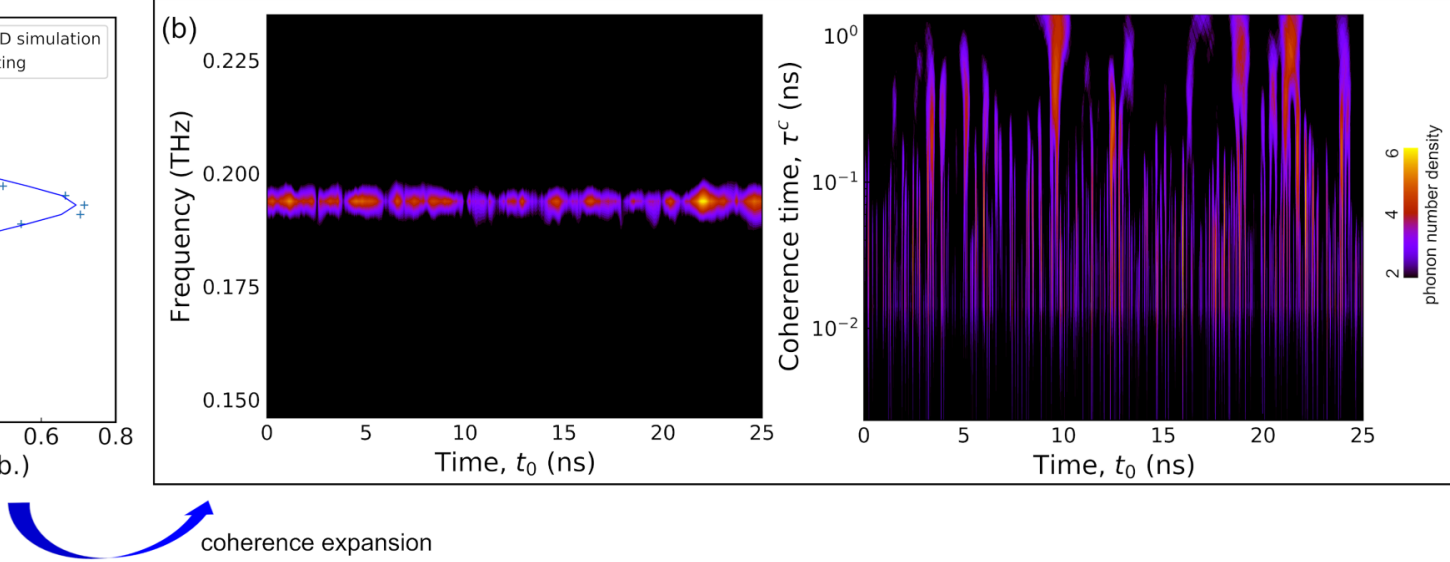

coherence expansion

FIG. 7. (a) SED simulation of mode $0.05 M$ ZA. The SED can be fitted by the Lorentz function (solid line), indicating the applying of SMRT approximation. (b) Wavelet transform results for mode $0.05 M$ ZA.

where $a_{\mathbf{k} s}$ and $a_{\mathbf{k} s}^{\dagger}$ respectively denote the phonon annihilation and creation operators. $V$ is the volume of the system, and $\omega_{\mathbf{k} s}$ and $v_{\mathbf{k} s}$ are the eigenfrequency and group velocity for mode $\mathbf{k} s$.

The terms in Eq. (C1) with $s \neq s^{\prime}$ contain contributions from modes with significantly different frequencies. Thus,
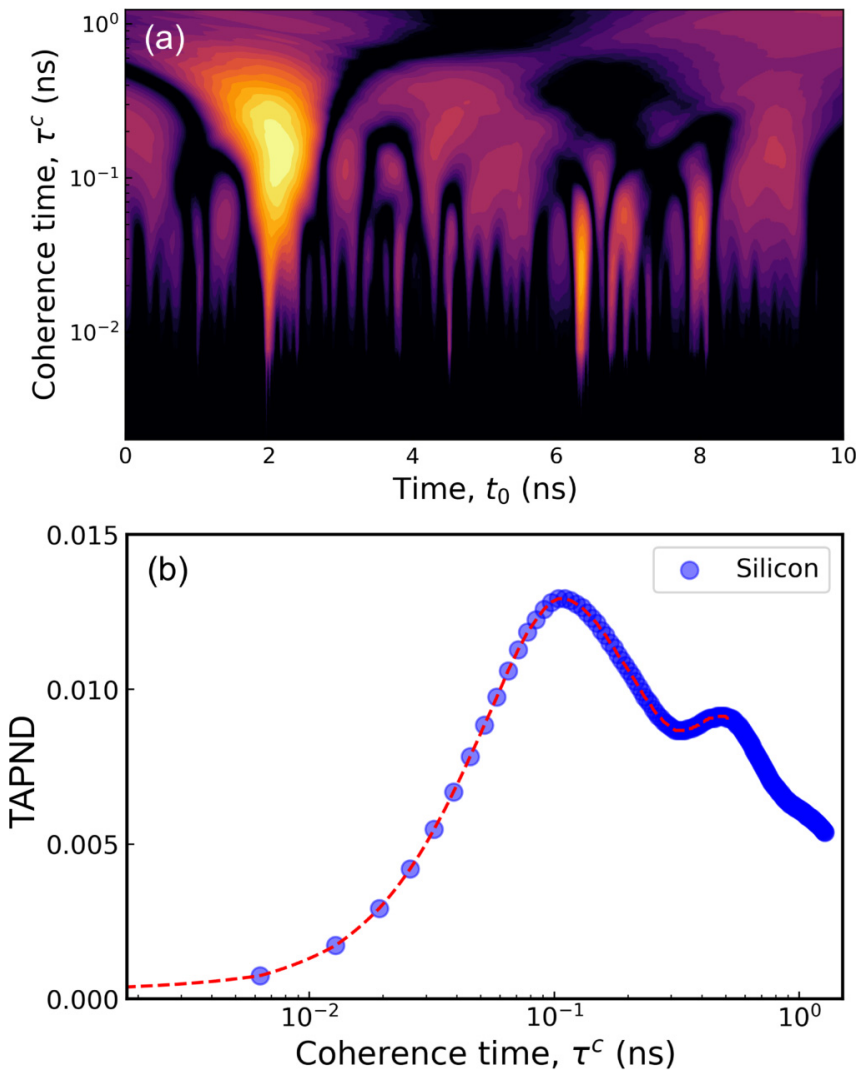

FIG. 8. Wavelet transform results in bulk silicon at room temperature. (a) Evolution time $\left(t_{0}\right)$ and coherence time $\left(\tau^{c}\right)$ dependent phonon number density for the $0.05 \mathrm{M}$ TA mode of silicon at room temperature. (b) Time-averaged phonon number density (TAPND) as a function of coherence time for the $0.05 \mathrm{M}$ TA mode at room temperature. their interferences are rapidly oscillating and become negligible after time averaging; accordingly these terms will be neglected. For the terms $s=s^{\prime}$ and $\mathbf{k} \neq \mathbf{k}^{\prime}$, the frequencies can be close and the resulting interferences can be kept in the further steps. The corresponding classical version of Eq. (C1) reads

$$
\mathbf{S}=\frac{1}{V} \sum_{\mathbf{k k}^{\prime}, s} p_{\mathbf{k} s} q_{\mathbf{k}^{\prime} s}^{*} \omega_{\mathbf{k} s} v_{\mathbf{k} s},
$$

where $p_{\mathbf{k} s}$ and $q_{\mathbf{k} s}$ are the modal momentum and velocity for mode $\mathbf{k} s$. They can be obtained as

$$
\begin{aligned}
& \mathbf{P}\left(\mathbf{x}_{i}\right)=N^{-1 / 2} \sum_{\mathbf{k} s} p_{\mathbf{k} s} \mathbf{e}_{\mathbf{k} s} e^{i \mathbf{k} \cdot \mathbf{x}_{i}}, \\
& \mathbf{Q}\left(\mathbf{x}_{i}\right)=N^{-1 / 2} \sum_{\mathbf{k} s} q_{\mathbf{k} s}^{*} \mathbf{e}_{\mathbf{k} s} e^{i \mathbf{k} \cdot \mathbf{x}_{i}} .
\end{aligned}
$$

Here, $\mathbf{P}\left(\mathbf{x}_{i}\right)$ and $\mathbf{Q}\left(\mathbf{x}_{i}\right)$ are respectively momentum and position operators of the $i$ th particle. $\mathbf{e}_{\mathbf{k} s}$ is the eigenvector of mode $\mathbf{k} s$, and $N$ is the total number of particles.

When introducing the time dependence into the energy flux, this latter can be expressed as a function of the products of the modal momentum $\left[p_{\mathbf{k} s}(t)\right]$ and displacement $\left[q_{\mathbf{k}^{\prime} s}(t)\right]$ fluctuations,

$$
\mathbf{S}(t)=\frac{1}{V} \sum_{\mathbf{k k}^{\prime}, s} p_{\mathbf{k} s}(t) q_{\mathbf{k}^{\prime} s}^{*}(t) \omega_{\mathbf{k} s} v_{\mathbf{k} s} .
$$

Considering that the phonon population can be quantified by $N_{\mathbf{k} s}$, the harmonic heat flux reduces to

$$
\mathbf{S}=\frac{1}{V} \sum_{\mathbf{k} s} N_{\mathbf{k} s} \hbar \omega_{\mathbf{k} s} v_{\mathbf{k} s} .
$$

The time dependent phonon number quantity $N_{\mathbf{k} s}(t)$ can be obtained as

$$
N_{\mathbf{k} s}(t)=\frac{1}{\hbar} \sum_{\mathbf{k}^{\prime}} p_{\mathbf{k} s}(t) q_{\mathbf{k}^{\prime} s}^{*}(t) .
$$

On this basis, we can study the fluctuations of the phonon number $N_{\mathbf{k} s}(t)$ from the dynamics of atomic motions. 
Moreover, including phonon-phonon scattering in the decay of normal mode coordinates yields [16]

$$
q_{\mathbf{k}^{\prime} s}(t)=q_{\mathbf{k}^{\prime} s}(0) e^{-\Gamma_{\mathbf{k}^{\prime} s} t-i \omega_{\mathbf{k}^{\prime} s} t},
$$

where $\Gamma_{\mathbf{k}^{\prime} s}$ is the linewidth of the mode $\mathbf{k}^{\prime} s$ corresponding to phonon-phonon scattering. The term $e^{-i \omega_{\mathbf{k}^{\prime} s} t}$ indicates the monochromaticity of the eigenmode, while the $e^{-\Gamma_{\mathbf{k}^{\prime} s} t}$ term corresponds to the decay of the vibration amplitude. The conjugate expression is defined as $q_{\mathbf{k}^{\prime} s}^{*}(t)=q_{\mathbf{k}^{\prime} s}^{*}(0) e^{-\Gamma_{\mathbf{k}^{\prime} s} t+i \omega_{\mathbf{k}^{\prime} s} t}$. This type of decay has been verified in inelastic neutron scattering measurements.

Accordingly, we assume that the time dependent modal momentum is

$$
p_{\mathbf{k} s}(t)=p_{\mathbf{k} s}(0) e^{-\Gamma_{\mathbf{k} s} t-i \omega_{\mathbf{k} s} t} .
$$

Consequently, the dynamic of the phonon number is obtained as

$$
N_{\mathbf{k} s}(t)=\sum_{\mathbf{k}^{\prime}} \xi_{\mathbf{k} \mathbf{k}^{\prime} s} e^{-\gamma_{\mathbf{k} \mathbf{k}^{\prime} s} t-i \Delta \omega_{\mathbf{k} \mathbf{k}^{\prime} s} t},
$$

where the variables in the above equation are $\xi_{\mathbf{k k}^{\prime} s}=$ $p_{\mathbf{k} s}(0) q_{\mathbf{k}^{\prime} s}^{*}(0) / \hbar, \gamma_{\mathbf{k} \mathbf{k}^{\prime} s}=\Gamma_{\mathbf{k} s}+\Gamma_{\mathbf{k}^{\prime} s}$, and $\Delta \omega_{\mathbf{k k}^{\prime} s}=\omega_{\mathbf{k} s}-\omega_{\mathbf{k}^{\prime} s}$.

It should also be noted that the summation over $\mathbf{k}^{\prime} s$ can be understood as the interference of $\mathbf{k}^{\prime} s$ and $\mathbf{k} s$ plane waves. During the phonon dynamics, the interference between different plane waves results in the generation of a phonon wave packet. In addition, we can assume that the frequency $\omega_{\mathbf{k}^{\prime} s}$ of the interfering plane waves is in the range of $\left[\omega_{\mathbf{k} s}-\frac{\Omega_{\mathbf{k} s}}{2}, \omega_{\mathbf{k} s}+\frac{\Omega_{\mathbf{k} s}}{2}\right]$. Moreover, to shift from a discrete summation to a continuous one, we also introduce the density of states for mode $\omega_{\mathbf{k}^{\prime} s}$, i.e., $g\left(\omega_{\mathbf{k}^{\prime} s}\right)$. Accordingly, Eq. (C9) can be rewritten in the integral form as

$$
N_{\mathbf{k} s}(t) \approx \int_{\omega_{\mathbf{k} s}-\left(\Omega_{\mathbf{k} s} / 2\right)}^{\omega_{\mathbf{k} s}+\left(\Omega_{\mathbf{k} s} / 2\right)} \xi_{\mathbf{k} \mathbf{k}^{\prime} s} e^{-\gamma_{\mathbf{k} \mathbf{k}^{\prime} s} t-i \Delta \omega_{\mathbf{k k}^{\prime} s} t} g\left(\omega_{\mathbf{k}^{\prime} s}\right) d \omega_{\mathbf{k}^{\prime} s} .
$$

On the other hand, because the interference occurs when plane-wave frequencies are close to each other, $\xi_{\mathbf{k} s s^{\prime}}, \gamma_{\mathbf{k} s s^{\prime}}$, and $g\left(\omega_{\mathbf{k}^{\prime} s}\right)$ do not change significantly. We can make a further step from Eq. (C10) as

$$
N_{\mathbf{k} s}(t) \approx \bar{\xi}_{\mathbf{k} s} e^{-\bar{\eta}_{\mathbf{k} s} t} \bar{g}\left(\omega_{\mathbf{k} s}\right) \int_{-\Omega_{\mathbf{k} s} / 2}^{+\Omega_{\mathbf{k s} s} / 2} e^{-i \Delta \omega_{\mathbf{k} \mathbf{k}^{\prime} s} t} d \Delta \omega_{\mathbf{k} \mathbf{k}^{\prime} s}
$$

where $\bar{\xi}_{\mathbf{k} s}, \bar{\gamma}_{\mathbf{k} s}$, and $\bar{g}\left(\omega_{\mathbf{k} s}\right)$ are respectively the averaged properties over one wave packet. The above integration yields a sinus cardinal (Sinc) function

$$
N_{\mathbf{k} s}(t)=2 \bar{\xi}_{\mathbf{k} s} e^{-\bar{\gamma}_{\mathbf{k} s} t} \bar{g}\left(\omega_{\mathbf{k} s}\right) \frac{\sin \pi \Omega_{\mathbf{k} s} t}{t},
$$

where the variables $\bar{\xi}_{\mathbf{k} s}, \bar{\gamma}_{\mathbf{k} s}, \bar{g}\left(\omega_{\mathbf{k} s}\right)$, and $\Omega_{\mathbf{k} s}$ are the specific properties of one type of wave packet. The term $e^{-\bar{\gamma}_{\mathrm{k} s} t}$ corresponds to the time decay of the phonon number, and the term $\frac{\sin \pi \Omega_{\mathrm{k} s} t}{t}$ indicates the shape of the phonon wave packet. Compared to a Gaussian shape, the sinus cardinal function has longer tails at the both ends, as shown in Fig. 9. Note that the time reference $t=0$ corresponds to the time of wavepacket maximum amplitude. This reference will disappear when deriving the autocorrelation of the phonon number. Nevertheless, the two types of wave packets almost have the same
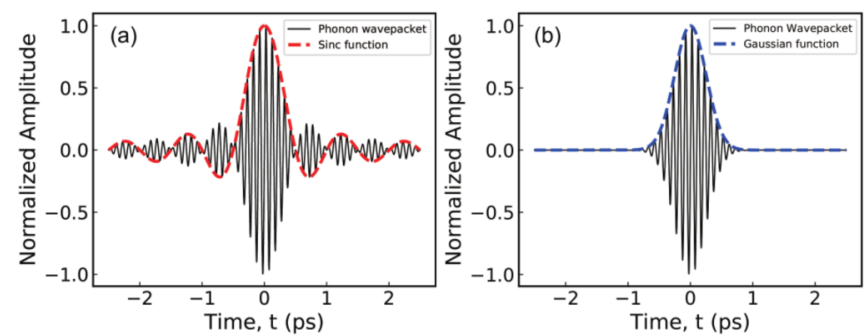

FIG. 9. The shape of the phonon wave packet. (a) The shape of the phonon wave packet in the form of a Sinc function, $e^{-i \omega t} \frac{\sin \pi \Omega t}{\pi \Omega t}$, with $\omega=10 \mathrm{THz}$ and $\Omega=1 \mathrm{THz}$. (b) The shape of the phonon wave packet in the form of a Gaussian function, $e^{-i \omega t} e^{-A \Omega^{2} t^{2}}$, with $\omega=10$ THz and $\Omega=1 \mathrm{THz}$. The parameter $A$ is set as 1.96 to make the Sinc and Gaussian functions have equal FWHM.

main amplitude at the center. Most importantly, Eq. (C12) also provides us with the information that at time $t$ one wave packet has emerged and is dynamically varying with the properties $\bar{\xi}_{\mathbf{k} s}, \bar{\gamma}_{\mathbf{k} s}, \bar{g}\left(\omega_{\mathbf{k} s}\right)$, and $\Omega_{\mathbf{k} s}$.

Because we consider the Gaussian basis in our wavelet transform method and because the Gaussian form will also be more tractable in the following, we would like to approximate the sinus cardinal with a Gaussian one. The principle is to fit both FWHM which define the temporal coherence. The resulting approximation reads

$$
N_{\mathbf{k} s}(t) \approx 4 \pi \Omega_{\mathbf{k} s} \bar{\xi}_{\mathbf{k} s} \bar{g}\left(\omega_{\mathbf{k} s}\right) e^{-\bar{\gamma}_{\mathbf{k} s} t} e^{-4 \ln 2 \Omega_{\mathbf{k s}}^{2} t^{2}}
$$

That is, at moment $t$, the phonon wave packet with frequency $\omega_{\mathbf{k} s}$ possesses inherent $\bar{\gamma}_{\mathbf{k} s}$ (approximately lifetime) and $\Omega_{\mathbf{k} s}(\sim 1 /$ coherence time). Equation (C13) models the apparition of a single wave packet. As illustrated in Fig. 1(c), a single wave packet indeed appears at a given time. To generalize Eq. (C13) to include the description of all wave packets, the phonon number shall be rewritten as a sum over all wave packets, each having their own peak at time $t_{0}$. However, this expression reduces back to Eq. (C13) at a given time.

\section{APPENDIX D: ANALYTICAL MODEL FOR PHONON DECAY}

As was discussed in the main text, the phonon dynamics can be investigated on the basis of the time autocorrelation of the phonon number. Starting from Eq. (C13), the autocorrelation function of the phonon wave packet defined by the physical quantities $\left(\bar{\gamma}_{\mathbf{k} s}, \Omega_{\mathbf{k} s}\right)$ is

$$
\left\langle N_{\mathbf{k} s}(t) N_{\mathbf{k} s}(0)\right\rangle=\left[4 \pi \Omega_{\mathbf{k} s} \bar{\xi}_{\mathbf{k} s} \bar{g}\left(\omega_{\mathbf{k} s}\right)\right]^{2}\left\langle\rho_{\mathbf{k} s}(t) \rho_{\mathbf{k} s}(0)\right\rangle,
$$

where $\rho_{\mathbf{k} s}(t)=e^{-\bar{\gamma}_{\mathbf{k} s} t-4 \ln 2 \Omega_{\mathbf{k} s}^{2} t^{2}}$. After several derivation steps, the normalized autocorrelation function can be obtained as

$$
\begin{aligned}
\frac{\left\langle N_{\mathbf{k} s}(t) N_{\mathbf{k} s}(0)\right\rangle}{\left\langle N_{\mathbf{k} s}(0) N_{\mathbf{k} s}(0)\right\rangle} & =\frac{\left\langle\rho_{\mathbf{k} s}(t) \rho_{\mathbf{k} s}(0)\right\rangle}{\left\langle\rho_{\mathbf{k} s}(0) \rho_{\mathbf{k} s}(0)\right\rangle} \\
& =e^{-(b / 2) t^{2}}\left(1-\sqrt{1-e^{-B^{2}}}\right),
\end{aligned}
$$


in which $B=\sqrt{\frac{b}{2}}\left(t+\frac{a}{b}\right), a=\bar{\gamma}_{\mathbf{k} s}$, and $b=4 \ln 2 \Omega_{\mathbf{k} s}^{2}$. We use the following series expansion to simplify the above equation:

$$
1-\sqrt{1-e^{-B^{2}}} \approx \frac{1}{2} e^{-B^{2}}-\frac{1}{8} e^{-2 B^{2}}+\frac{1}{16} e^{-3 B^{2}}-\frac{5}{128} e^{-4 B^{2}} .
$$

We now limit the expansion to the first order with $1-$ $\sqrt{1-e^{-B^{2}}} \approx \frac{1}{2} e^{-B^{2}}$ by considering that $B>1$ in which the first-order expansion is accurate. Thus, the normalized autocorrelation function is simplified as

$$
\frac{\left\langle N_{\mathbf{k} s}(t) N_{\mathbf{k} s}(0)\right\rangle}{\left\langle N_{\mathbf{k} s}(0) N_{\mathbf{k} s}(0)\right\rangle} \approx e^{-(b / 2) t^{2}} e^{-B^{2}}=e^{-\bar{\gamma}_{\mathbf{k} s} t} e^{-4 \ln 2 \Omega_{\mathbf{k} s}^{2} t^{2}} .
$$

Previously, it has been demonstrated that the linewidth $\bar{\gamma}_{\mathbf{k} s}$ for mode $\mathbf{k} s$ is related to the lifetime $\tau_{\mathbf{k} s}^{l}$ as follows:

$$
\tau_{\mathbf{k} s}^{l}=\frac{1}{2 \bar{\gamma}_{\mathbf{k} s}} \text {. }
$$

In this work, we define $\tau_{\mathbf{k} s}^{c}$ as the FWHM of the wave packet. Therefore, by redefining the term $e^{-4 \ln 2 \Omega_{\mathbf{k s}}^{2} t^{2}}$, Eq. (D4) can be rewritten as

$$
\frac{\left\langle N_{\mathbf{k} s}(t) N_{\mathbf{k} s}(0)\right\rangle}{\left\langle N_{\mathbf{k} s}(0) N_{\mathbf{k} s}(0)\right\rangle}=e^{-t / 2 \tau_{\mathbf{k s} s}^{l}} e^{-4 \ln 2\left(t^{2} / \tau_{\mathbf{k s}}^{c}\right)} .
$$

In the above equation the exponential part, $e^{-t / 2 \tau_{\mathbf{k s}}^{l}}$, results from phonon-phonon scattering. In contrast, the Gaussian part, $e^{-4 \ln 2\left(t^{2} / \tau_{\mathbf{k s}}^{c}\right)}$, corresponds to the effect of plane-wave interferences and the formation of wave packets with a finite duration. Considering the unfolding of the phonon number, the autocorrelation function for mode $\mathbf{k} s$ along coherence time $\tau_{\mathbf{k} s}^{c}, C\left(t, \tau_{\mathbf{k} s}^{c}\right)$, can be inferred as

$$
C\left(t, \tau_{\mathbf{k} s}^{c}\right)=e^{-t / 2 \tau_{\mathbf{k s}}^{l}} e^{\left.-4 \ln 2\left(t^{2} / \tau_{\mathbf{k s}}^{c}\right)^{2}\right)} .
$$

[1] R. Peierls, Zur kinetischen theorie der wärmeleitung in kristallen, Ann. Phys. 395, 1055 (1929).

[2] J. M. Ziman, Electrons and Phonons: The Theory of Transport Phenomena in Solids (Oxford University Press, New York, 2001).

[3] M. N. Luckyanova, J. Garg, K. Esfarjani, A. Jandl, M. T. Bulsara, A. J. Schmidt, A. J. Minnich, S. Chen, M. S. Dresselhaus, and Z. Ren, Coherent phonon heat conduction in superlattices, Science 338, 936 (2012).

[4] J. Ravichandran, A. K. Yadav, R. Cheaito, P. B. Rossen, A. Soukiassian, S. J. Suresha, J. C. Duda, B. M. Foley, C. H. Lee, Y. Zhu, A. W. Lichtenberger, J. E. Moore, D. A. Muller, D. G. Schlom, P. E. Hopkins, A. Majumdar, R. Ramesh, and M. A. Zurbuchen, Crossover from incoherent to coherent phonon scattering in epitaxial oxide superlattices, Nat. Mater. 13, 168 (2014).

[5] J. Maire, R. Anufriev, R. Yanagisawa, A. Ramiere, S. Volz, and $\mathrm{M}$. Nomura, Heat conduction tuning by wave nature of phonons, Sci. Adv. 3, e1700027 (2017).

[6] S. Hu, Z. Zhang, P. Jiang, W. Ren, C. Yu, J. Shiomi, and J. Chen, Disorder limits the coherent phonon transport in twodimensional phononic crystal structures, Nanoscale 11, 11839 (2019).

[7] S. Alaie, D. F. Goettler, M. Su, Z. C. Leseman, C. M. Reinke, and I. El-Kady, Thermal transport in phononic crystals and the observation of coherent phonon scattering at room temperature, Nat. Commun. 6, 7228 (2015).

[8] B. Latour and Y. Chalopin, Distinguishing between spatial coherence and temporal coherence of phonons, Phys. Rev. B 95, 214310 (2017).

[9] H. Han, B. Li, S. Volz, and Y. A. Kosevich, Ultracompact Interference Phonon Nanocapacitor for Storage and Lasing of Coherent Terahertz Lattice Waves, Phys. Rev. Lett. 114, 145501 (2015).

[10] Z. Zhang, S. Hu, Q. Xi, T. Nakayama, S. Volz, J. Chen, and B. $\mathrm{Li}$, Tunable phonon nanocapacitor built by carbon schwarzite based host-guest system, Phys. Rev. B 101, 081402(R) (2020).

[11] A. Cepellotti and N. Marzari, Thermal Transport in Crystals as a Kinetic Theory of Relaxons, Phys. Rev. X 6, 041013 (2016).
[12] Y. Wang, H. Huang, and X. Ruan, Decomposition of coherent and incoherent phonon conduction in superlattices and random multilayers, Phys. Rev. B 90, 165406 (2014).

[13] S. Hu, Z. Zhang, P. Jiang, J. Chen, S. Volz, M. Nomura, and B. $\mathrm{Li}$, Randomness-induced phonon localization in graphene heat conduction, J. Phys. Chem. Lett. 9, 3959 (2018).

[14] B. Latour, S. Volz, and Y. Chalopin, Microscopic description of thermal-phonon coherence: From coherent transport to diffuse interface scattering in superlattices, Phys. Rev. B 90, 014307 (2014).

[15] G. Xie, D. Ding, and G. Zhang, Phonon coherence and its effect on thermal conductivity of nanostructures, Adv. Phys.: X 3, 720 (2018).

[16] A. J. C. Ladd, B. Moran, and W. G. Hoover, Lattice thermal conductivity: A comparison of molecular dynamics and anharmonic lattice dynamics, Phys. Rev. B 34, 5058 (1986).

[17] A. Ward, D. A. Broido, D. A. Stewart, and G. Deinzer, Ab initio theory of the lattice thermal conductivity in diamond, Phys. Rev. B 80, 125203 (2009).

[18] J. E. Turney, E. S. Landry, A. J. H. McGaughey, and C. H. Amon, Predicting phonon properties and thermal conductivity from anharmonic lattice dynamics calculations and molecular dynamics simulations, Phys. Rev. B 79, 064301 (2009).

[19] A. Glensk, B. Grabowski, T. Hickel, J. Neugebauer, J. Neuhaus, K. Hradil, W. Petry, and M. Leitner, Phonon Lifetimes throughout the Brillouin Zone at Elevated Temperatures from Experiment and Ab Initio, Phys. Rev. Lett. 123, 235501 (2019).

[20] J. Cuffe, O. Ristow, E. Chavez, A. Shchepetov, P. O. Chapuis, F. Alzina, M. Hettich, M. Prunnila, J. Ahopelto, T. Dekorsy, and C. M. Sotomayor Torres, Lifetimes of Confined Acoustic Phonons in Ultrathin Silicon Membranes, Phys. Rev. Lett. 110, 095503 (2013).

[21] M. Simoncelli, N. Marzari, and F. Mauri, Unified theory of thermal transport in crystals and glasses, Nat. Phys. 15, 809 (2019).

[22] L. Isaeva, G. Barbalinardo, D. Donadio, and S. Baroni, Modeling heat transport in crystals and glasses from a unified lattice-dynamical approach, Nat. Commun. 10, 3853 (2019). 
[23] J. M. Larkin, J. E. Turney, A. D. Massicotte, C. H. Amon, and A. J. H. McGaughey, Comparison and evaluation of spectral energy methods for predicting phonon properties, J. Comput. Theor. Nanosci. 11, 249 (2014).

[24] M. T. Dove, Introduction to Lattice Dynamics (Cambridge University Press, Cambridge, UK, 1993), Vol. 4.

[25] P. K. Schelling, S. R. Phillpot, and P. Keblinski, Phonon wavepacket dynamics at semiconductor interfaces by moleculardynamics simulation, Appl. Phys. Lett. 80, 2484 (2002).

[26] P. Y. Yu and M. Cardona, Fundamentals of Semiconductors: Physics and Materials Properties (Springer, New York, 1996).

[27] J. P. Wolfe, Imaging Phonons: Acoustic Wave Propagation in Solids (Cambridge University Press, Cambridge, UK, 2005).

[28] G. P. Srivastava, The Physics of Phonons (Routledge, New York, 2019).

[29] C. H. Baker, D. A. Jordan, and P. M. Norris, Application of the wavelet transform to nanoscale thermal transport, Phys. Rev. B 86, 104306 (2012).

[30] J. Shiomi and S. Maruyama, Non-Fourier heat conduction in a single-walled carbon nanotube: Classical molecular dynamics simulations, Phys. Rev. B 73, 205420 (2006).

[31] S. Srinivasan and G. Balasubramanian, Reduced thermal transport in the graphene/MosS $\mathrm{S}_{2}$ /graphene heterostructure: A comparison with freestanding monolayers, Langmuir 34, 3326 (2018).

[32] Y. J. Han and P. G. Klemens, Anharmonic thermal resistivity of dielectric crystals at low temperatures, Phys. Rev. B 48, 6033 (1993).
[33] D. Snoke, Solid State Physics: Essential Concepts (Cambridge University Press, Cambridge, UK, 2020).

[34] D. L. Nika, E. P. Pokatilov, A. S. Askerov, and A. A. Balandin, Phonon thermal conduction in graphene: Role of umklapp and edge roughness scattering, Phys. Rev. B 79, 155413 (2009).

[35] L. Lindsay and D. A. Broido, Optimized Tersoff and Brenner empirical potential parameters for lattice dynamics and phonon thermal transport in carbon nanotubes and graphene, Phys. Rev. B 81, 205441 (2010).

[36] Z. Fan, W. Chen, V. Vierimaa, and A. Harju, Efficient molecular dynamics simulations with many-body potentials on graphics processing units, Comput. Phys. Commun. 218, 10 (2017).

[37] R. J. Hardy, Energy-flux operator for a lattice, Phys. Rev. 132, 168 (1963).

[38] A. Cepellotti, G. Fugallo, L. Paulatto, M. Lazzeri, F. Mauri, and N. Marzari, Phonon hydrodynamics in two-dimensional materials, Nat. Commun. 6, 6400 (2015).

[39] D. C. Wallace, Thermodynamics of crystals, Am. J. Phys. 40, 1718 (1972).

[40] G. Chen, Nanoscale Energy Transport and Conversion: A Parallel Treatment of Electrons, Molecules, Phonons, and Photons (Oxford University Press, New York, 2005).

[41] A. J. H. McGaughey and M. Kaviany, Quantitative validation of the Boltzmann transport equation phonon thermal conductivity model under the single-mode relaxation time approximation, Phys. Rev. B 69, 094303 (2004).

[42] Z. Zhang, Y. Ouyang, Y. Cheng, J. Chen, N. Li, and G. Zhang, Size-dependent phononic thermal transport in low-dimensional nanomaterials, Phys. Rep. 860, 1 (2020). 